\title{
ALGEBRAIC MATCHING THEORY
}

\author{
C. D. Godsil ${ }^{1}$ \\ Department of Combinatorics and Optimization \\ University of Waterloo \\ Waterloo, Ontario \\ Canada N2L 3G1 \\ chris@bilby.uwaterloo.ca
}

Submitted: July 6, 1994; Accepted: April 11, 1995.

\begin{abstract}
The number of vertices missed by a maximum matching in a graph $G$ is the multiplicity of zero as a root of the matchings polynomial $\mu(G, x)$ of $G$, and hence many results in matching theory can be expressed in terms of this multiplicity. Thus, if $\operatorname{mult}(\theta, G)$ denotes the multiplicity of $\theta$ as a zero of $\mu(G, x)$, then Gallai's lemma is equivalent to the assertion that if $\operatorname{mult}(\theta, G \backslash u)<$ $\operatorname{mult}(\theta, G)$ for each vertex $u$ of $G$, then $\operatorname{mult}(\theta, G)=1$.

This paper extends a number of results in matching theory to results concerning mult $(\theta, G)$, where $\theta$ is not necessarily zero. If $P$ is a path in $G$ then $G \backslash P$ denotes the graph got by deleting the vertices of $P$ from $G$. We prove that $\operatorname{mult}(\theta, G \backslash P) \geq \operatorname{mult}(\theta, G)-1$, and we say $P$ is $\theta$-essential when equality holds. We show that if, all paths in $G$ are $\theta$-essential, then $\operatorname{mult}(\theta, G)=1$. We define $G$ to be $\theta$-critical if all vertices in $G$ are $\theta$-essential and $\operatorname{mult}(\theta, G)=1$. We prove that if $\operatorname{mult}(\theta, G)=k$ then there is an induced subgraph $H$ with exactly $k$ $\theta$-critical components, and the vertices in $G \backslash H$ are covered by $k$ disjoint paths.
\end{abstract}

AMS Classification Numbers: 05C70, 05E99

1 Support from grant OGP0009439 of the National Sciences and Engineering Council of Canada is gratefully acknowledged. 


\section{Introduction}

A $k$-matching in a graph $G$ is a matching with exactly $k$ edges and the number of $k$ matchings in $G$ is denoted by by $p(G, k)$. If $n=|V(G)|$ we define the matchings polynomial $\mu(G, x)$ by

$$
\mu(G, x):=\sum_{k \geq 0}(-1)^{k} p(G, k) x^{n-2 k} .
$$

(Here $p(G, 0)=1$.) By way of example, the matchings polynomial of the path on four vertices is $x^{4}-3 x^{2}+1$. The matchings polynomial is related to the characteristic polynomial $\phi(G, x)$ of $G$, which is defined to be the characteristic polynomial of the adjacency matrix of $G$. In particular $\phi(G, x)=\mu(G, x)$ if and only if $G$ is a forest [4: Corollary 4.2]. Also the matchings polynomial of any connected graph is a factor of the characteristic polynomial of some tree. (For this, see Theorem 2.2 below.)

Let mult $(\theta, G)$ denote the multiplicity of $\theta$ as a zero of $\mu(G, x)$. If $\theta=0$ then mult $(\theta, G)$ is the number of vertices in $G$ missed by a maximum matching. Consequently many classical results in the theory of matchings provide information related to mult $(0, G)$. We refer in particular to Gallai's lemma and the Edmonds-Gallai structure theorem, which we now discuss briefly.

A vertex $u$ of $G$ is $\theta$-essential if mult $(\theta, G \backslash u)<\operatorname{mult}(\theta, G)$. So a vertex is 0-essential if and only if it is missed by some maximum matching of $G$. Gallai's lemma is the assertion that if $G$ is connected, $\theta=0$ and every vertex is $\theta$-essential then mult $(\theta, G)=1$. (A more traditional expression of this result is given in [8: §3.1].) A vertex is $\theta$-special if it is not $\theta$-essential but has a neighbour which is $\theta$-essential. The Edmonds-Gallai structure in large part reduces to the assertion that if $\theta=0$ and $v$ is a $\theta$-special vertex in $G$ then a vertex $u$ is $\theta$-essential in $G$ and if and only if it is $\theta$-essential in $G \backslash v$. (For more information, see [8: §3.2].) One aim of the present paper is to investigate the extent to which these results are true when $\theta \neq 0$.

There is a second source of motivation for our work. Heilman and Lieb proved that if $G$ has a Hamilton path then all zeros of $\mu(G, x)$ are simple. (This is an easy consequence of Corollary 2.5 below.) Since all known vertex-transitive graphs have Hamilton paths we are lead to ask whether there is a vertex-transitive graph $G$ such that $\mu(G, x)$ has a multiple zero. As we will see, it is easy to show that if $\theta$ is a zero of $\mu(G, x)$ and $G$ is vertex-transitive then every vertex of $G$ is $\theta$-essential. Hence, if we could prove Gallai's lemma for general zeros of the matchings polynomial, we would have a negative answer to this question. 


\section{Identities}

The first result provides the basic properties of the matchings polynomial $\mu(G, x)$. We write $u \sim v$ to denote that the vertex $u$ is adjacent to the vertex $v$. For the details see, for example, [6: Theorem 1.1].

2.1 Theorem. The matchings polynomial satisfies the following identities:

(a) $\mu(G \cup H, x)=\mu(G, x) \mu(H, x)$,

(b) $\mu(G, x)=\mu(G \backslash e, x)-\mu(G \backslash u v, x)$ if $e=\{u, v\}$ is an edge of $G$,

(c) $\mu(G, x)=x \mu(G \backslash u, x)-\sum_{i \sim u} \mu(G \backslash u i, x)$, if $u \in V(G)$,

(d) $\frac{d}{d x} \mu(G, x)=\sum_{i \in V(G)} \mu(G \backslash i, x)$.

Let $G$ be a graph with a vertex $u$. By $\mathcal{P}(u)$ we denote the set of paths in $G$ which start at $u$. The path tree $T(G, u)$ of $G$ relative to $u$ has $\mathcal{P}(u)$ as its vertex set, and two paths are adjacent if one is a maximal proper subpath of the other. Note that each path in $\mathcal{P}(u)$ determines a path starting with $u$ in $T(G, u)$ and with same length. We will usually denote them by the same symbol. The following result is taken from [6: Theorem 6.1.1].

2.2 Theorem. Let $u$ be a vertex in the graph $G$ and let $T=T(G, u)$ be the path tree of $G$ with respect to $u$. Then

$$
\frac{\mu(G \backslash u, x)}{\mu(G, x)}=\frac{\mu(T \backslash u, x)}{\mu(T, x)}
$$

and, if $G$ is connected, then $\mu(G, x)$ divides $\mu(T, x)$.

Because the matchings polynomial of a tree is equal to the characteristic polynomial of its adjacency matrix, its zeros are real; consequently Theorem 2.2 implies that the zeros of the matchings polynomial of $G$ are real, and also that they are interlaced by the zeros of $\mu(G \backslash u, x)$, for any vertex $u$. (By interlace, we mean that, between any two zeros of $\mu(G, x)$, there is a zero of $\mu(G \backslash u, x)$. This implies in particular that the multiplicity of a zero $\theta$ in $\mu(G, x)$ and $\mu(G \backslash u, x)$ can differ by at most one.) For a more extensive discussion of these matters, see [6: $\S 6.1]$.

We will need a strengthening of the first claim in Theorem 2.2 .

2.3 Corollary. Let $u$ be a vertex in the graph $G$ and let $T=T(G, u)$ be the path tree of $G$ with respect to $u$. If $P \in \mathcal{P}(u)$ then

$$
\frac{\mu(G \backslash P, x)}{\mu(G, x)}=\frac{\mu(T \backslash P, x)}{\mu(T, x)} .
$$


Proof. We proceed by induction on the number of vertices in $P$. If $P$ has only one vertex, we appeal to the theorem. Suppose then that $P$ has at least two vertices in it, and that $v$ is the end vertex of $P$ other than $u$. Let $Q$ be the path $P \backslash v$ and let $H$ denote $G \backslash Q$. Then

$$
\frac{\mu(G \backslash P, x)}{\mu(G, x)}=\frac{\mu(G \backslash P, x)}{\mu(G \backslash Q, x)} \frac{\mu(G \backslash Q, x)}{\mu(G, x)}=\frac{\mu(T(H, v) \backslash v, x)}{\mu(T(H, v), x)} \frac{\mu(T \backslash Q, x)}{\mu(T, x)},
$$

where the second equality follows by induction. Now $T(H, v)$ is one component of $T(G, u) \backslash$ $Q$, and if we delete the vertex $v$ from this component from $T(G, u) \backslash Q$, the graph that results is $T(G, u) \backslash P$. Consequently

$$
\frac{\mu(T(H, v) \backslash v, x)}{\mu(T(H, v), x)}=\frac{\mu(T \backslash P, x)}{\mu(T \backslash Q, x)} .
$$

The results follows immediately from this.

Let $\mathcal{P}(u, v)$ denote the set of paths in $G$ which start at $u$ and finish at $v$. The following result will be one of our main tools. It is a special case of [7: Theorem 6.3].

2.4 Lemma (Heilmann and Lieb). Let $u$ and $v$ be vertices in the graph $G$. Then

$$
\mu(G \backslash u, x) \mu(G \backslash v, x)-\mu(G, x) \mu(G \backslash u v, x)=\sum_{P \in \mathcal{P}(u, v)} \mu(G \backslash P, x)^{2} .
$$

This lemma has a number of important consequences. In [5: Section 4] it is used to show that mult $(\theta, G)$ is a lower bound on the number of paths needed to cover the vertices of $G$, and that the number of distinct zeros of $\mu(G, x)$ is an upper bound on the length of a longest path. For our immediate purposes, the following will be the most useful.

2.5 Corollary. If $P$ is a path in the graph $G$ then $\mu(G \backslash P, x) / \mu(G, x)$ has only simple poles. In other words, for any zero $\theta$ of $\mu(G, x)$ we have

$$
\operatorname{mult}(\theta, G \backslash P) \geq \operatorname{mult}(\theta, G)-1 \text {. }
$$

Proof. Suppose $k=\operatorname{mult}(\theta, G)$. Then, by interlacing, $\operatorname{mult}(\theta, G \backslash u) \geq k-1$ for any vertex $u$ of $G$ and $\operatorname{mult}(\theta, G \backslash u v) \geq k-2$. Hence the multiplicity of $\theta$ as a zero of

$$
\mu(G \backslash u, x) \mu(G \backslash v, x)-\mu(G, x) \mu(G \backslash u v, x)
$$

is at least $2 k-2$. It follows from Lemma 2.4 that $\operatorname{mult}(\theta, G \backslash P) \geq k-1$ for any path $P$ in $\mathcal{P}(u, v)$. 


\section{Essential Vertices and Paths}

Let $\theta$ be a zero of $\mu(G, x)$. A path $P$ of $G$ is $\theta$-essential if mult $(\theta, G \backslash P)<\operatorname{mult}(\theta, G)$. (We will often be concerned with the case where $P$ is a single vertex.) A vertex is $\theta$-special if it is not $\theta$-essential and is adjacent to an $\theta$-essential vertex. A graph is $\theta$-primitive if and only if every vertex is $\theta$-essential and it is $\theta$-critical if it is $\theta$-primitive and $\operatorname{mult}(\theta, G)=1$. (When $\theta$ is determined by the context we will often drop the prefix ' $\theta$-' from these expressions.) If $\theta=0$ then a $\theta$-critical graph is the same thing as a factor-critical graph.

The next result implies that a vertex-transitive graph is $\theta$-primitive for any zero $\theta$ of its matchings polynomial.

3.1 Lemma. Any graph has at least one essential vertex.

Proof. Let $\theta$ be a zero of $\mu(G, x)$ with multiplicity $k$. Then $\theta$ has multiplicity $k-1$ as a zero of $\mu^{\prime}(G, x)$. Since

$$
\mu^{\prime}(G, x)=\sum_{u \in V(G)} \mu(G \backslash u, x)
$$

we see that if $\operatorname{mult}(\theta, G \backslash u) \geq k$ for all vertices $u$ of $G$ then $\theta$ must have multiplicity at least $k$ as a zero of $\mu^{\prime}(G, x)$.

3.2 Lemma. If $\theta \neq 0$ then any $\theta$-essential vertex $u$ has a neighbour $v$ such that the path $u v$ is essential.

Proof. Assume $\theta \neq 0$ and let $u$ be a $\theta$-essential vertex. Since

$$
\mu(G, x)=x \mu(G \backslash u, x)-\sum_{i \sim u} \mu(G \backslash u i, x)
$$

we see that if $\operatorname{mult}(\theta, G \backslash u i) \geq \operatorname{mult}(\theta, G)$ for all neighbours $i$ of $u$ then $\operatorname{mult}(\theta, G \backslash u) \geq$ $\operatorname{mult}(\theta, G)$.

Note that the vertex $v$ is not essential in $G \backslash u$. However it follows from the next lemma that the vertex $v$ in the above lemma must be essential in $G$; accordingly if $\theta \neq 0$ then any essential vertex must have an essential neighbour. 
THE ELECTRonic JOURnAl of COMBinAtorics 2 (1995), \#R8

3.3 Lemma. If $v$ is not an essential vertex of $G$ then no path with $v$ as an end-vertex is essential.

Proof. Assume $k=\operatorname{mult}(\theta, G)$. If $v$ is not essential then $\operatorname{mult}(\theta, G \backslash v) \geq k$ and so, for any vertex $u$ not equal to $v$, the multiplicity of $\theta$ as a zero of

$$
\mu(G \backslash u, x) \mu(G \backslash v, x)-\mu(G, x) \mu(G \backslash u v, x)
$$

is at least $2 k-1$. By Lemma 2.4 we deduce that it is at least $2 k$ and that $\operatorname{mult}(\theta, G \backslash P) \geq k$ for all paths $P$ in $\mathcal{P}(v)$.

We now need some more notation. Suppose that $G$ is a graph and $\theta$ is a zero of $\mu(G, x)$ with positive multiplicity $k$. A vertex $u$ of $G$ is $\theta$-positive if $\operatorname{mult}(\theta, G \backslash u)=k+1$ and $\theta$-neutral if $\operatorname{mult}(\theta, G \backslash u)=k$. (The 'negative' vertices will still be referred to as essential.) Note that, by interlacing, $\operatorname{mult}(\theta, G \backslash u)$ cannot be greater than $k+1$.

3.4 Lemma. Let $G$ be a graph and $u$ a vertex in $G$ which is not essential. Then $u$ is positive in $G$ if and only if some neighbour of it is essential in $G \backslash u$.

Proof. From Theorem 2.1(c) we have

$$
\mu(G, x)=x \mu(G \backslash u, x)-\sum_{i \sim u} \mu(G \backslash u i, x) .
$$

If $\operatorname{mult}(\theta, G \backslash u)=k+1$ and $\operatorname{mult}(\theta, G \backslash u i) \geq k+1$ for all neighbours $i$ of $u$ then it follows that $\operatorname{mult}(\theta, G) \geq k+1$ and $u$ is not positive.

On the other hand, suppose $u$ is not essential in $G$ and $v$ is a neighbour of $u$ which is essential in $G \backslash u$. From the previous lemma we see that the path $u v$ is not essential and thus $\operatorname{mult}(\theta, G \backslash u v) \geq \operatorname{mult}(\theta, G)$. As $v$ is essential in $G \backslash u$ it follows that $\operatorname{mult}(\theta, G \backslash u)>$ $\operatorname{mult}(\theta, G)$.

We say that $S$ is an extremal subtree of the tree $T$ if $S$ is a component of $T \backslash v$ for some vertex $v$ of $G$.

3.5 Lemma. Let $S$ be an extremal subtree of $T$ that is inclusion-minimal subject to the condition that mult $(\theta, S) \neq 0$, and let $v$ be the vertex of $T$ such that $S$ is a component of $T \backslash v$. Then $v$ is $\theta$-positive in $T$.

Proof. Let $u$ be the vertex of $S$ adjacent to $v$ and let $e$ be the edge $\{u, v\}$. Then $T \backslash e$ has exactly two components, one of which is $S$. Denote the other by $R$. 
By hypothesis mult $\left(\theta, S^{\prime}\right)=0$ for any component $S^{\prime}$ of $S \backslash u$, therefore mult $(\theta, S \backslash u)=0$ by Theorem 2.1(a) and so $u$ is essential in $S$. Since $S$ is a component of $T \backslash v$ it follows that $u$ is essential in $T \backslash v$. If we can show that $v$ is not essential then $v$ must be positive in $T$, by the previous lemma.

Suppose $\operatorname{mult}(\theta, T)=m$. By interlacing $\operatorname{mult}(\theta, T \backslash u) \geq m-1$ and, as

$$
\operatorname{mult}(\theta, T \backslash u)=\operatorname{mult}(\theta, R)+\operatorname{mult}(\theta, S \backslash u)=\operatorname{mult}(\theta, R),
$$

we find that mult $(\theta, R) \geq m-1$. By parts (a) and (b) of Theorem 2.1 we have

$$
\mu(T, x)=\mu(R, x) \mu(S, x)-\mu(R \backslash v, x) \mu(S \backslash u, x)
$$

and so, since the multiplicity of $\theta$ as a zero of $\mu(R, x) \mu(S, x)$ is at least $m$, we deduce that the multiplicity of $\theta$ as a zero of $\mu(R \backslash v, x) \mu(S \backslash u, x)$ is at least $m$. Since $\operatorname{mult}(\theta, S \backslash u)=0$, it follows that $\operatorname{mult}(\theta, R \backslash v) \geq m$. On the other hand

$$
\operatorname{mult}(\theta, T \backslash v)=\operatorname{mult}(\theta, R \backslash v)+\operatorname{mult}(\theta, S)=\operatorname{mult}(\theta, R \backslash v)+1,
$$

therefore $\operatorname{mult}(\theta, T \backslash v) \geq m+1$ and $v$ is positive in $T$.

3.6 Corollary (Neumaier). Let $T$ be a tree and let $\theta$ be a zero of $\mu(T, x)$. The following assertions are equivalent:

(a) $\operatorname{mult}(\theta, S)=0$ for all extremal subtrees of $T$,

(b) $T$ is $\theta$-critical,

(c) $T$ is $\theta$-primitive.

Proof. Since $T \backslash v$ is a disjoint union of extremal subtrees for any vertex $v$ in $T$, we see that if (a) holds then mult $(\theta, T \backslash v)=0$ for any vertex $v$. Hence $T$ is $\theta$-critical and therefore it is also $\theta$-primitive. If $T$ is $\theta$-primitive then no vertex in $T$ is $\theta$-positive, whence Lemma 3.5 implies that (a) holds.

Corollary 3.6 combines Theorem 3.1 and Corollary 3.3 from [9]. Note that the equivalence of (b) and (c) when $\theta=0$ is Gallai's lemma for trees.

3.7 Lemma. Let $G$ be a connected graph. If $u \in V(G)$ and all paths in $G$ starting at $u$ are essential then $G$ is critical.

Proof. If all paths in $\mathcal{P}(u)$ are essential then Lemma 3.3 implies that all vertices in $G$ are essential. Hence $G$ is primitive, and it only remains for us to show that $\operatorname{mult}(\theta, G)=1$. 
Let $T=T(G, u)$ be the path tree of $G$ relative to $u$. From Theorem 2.2 we see that a path $P$ from $\mathcal{P}(u)$ is essential in $G$ if and only if it is essential in $T$. So our hypothesis implies that all paths in $T$ which start at $u$ are essential, whence Lemma 3.3 yields that all vertices in $T$ are essential. Hence $T$ is $\theta$-primitive and therefore, by Corollary 3.6, $\theta$ is a simple zero of $\mu(T, x)$. Using Theorem 2.2 again we deduce that mult $(\theta, G)=1$.

3.8 Lemma. If $u$ and $v$ are essential vertices in $G$ and $v$ is not essential in $G \backslash u$ then there is a $\theta$-essential path in $\mathcal{P}(u, v)$.

Proof. Assume mult $(\theta, G)=k$. Our hypotheses imply that mult $(\theta, G \backslash u v) \geq k-1$. If no path in $\mathcal{P}(u, v)$ is essential then, by Lemma 2.4, the multiplicity of $\theta$ as a zero of

$$
\mu(G \backslash u, x) \mu(G \backslash v, x)-\mu(G, x) \mu(G \backslash u v, x)
$$

is at least $2 k$. Since $\theta$ has multiplicity $2 k-1$ as a zero of $\mu(G, x) \mu(G \backslash u v, x)$ it must also have multiplicity at least $2 k-1$ as a zero of $\mu(G \backslash u, x) \mu(G \backslash v, x)$. Hence $u$ and $v$ cannot both be essential.

If $u$ and $v$ are essential in $G$ then $v$ is essential in $G \backslash u$ if and only if $u$ is essential in $G \backslash v$. Thus the hypothesis of Lemma 3.8 is symmetric in $u$ and $v$, despite appearances.

3.9 Corollary. Let $G$ be a tree, let $\theta$ be a zero of $\mu(G, x)$ and let $u$ be a vertex in $G$. Then all paths in $\mathcal{P}(u)$ are essential if and only if all vertices in $G$ are essential.

Proof. It follows from Lemma 3.3 that if all paths in $\mathcal{P}(u)$ are essential then all vertices in $G$ are essential. Suppose conversely that all vertices in $G$ are essential. By Corollary 3.6 it follows that $\operatorname{mult}(\theta, G)=1$. Hence the hypotheses of Lemma 3.8 are satisfied by any two vertices in $G$, and so any two vertices are joined by an essential path. Since $G$ is a tree the path joining any two vertices is unique and therefore all paths in $\mathcal{P}(u)$ are essential. 


\section{Structure Theorems}

We now apply the machinery we have developed in the previous section.

4.1 Lemma (De Caen [2]). Let $u$ and $v$ be adjacent vertices in a bipartite graph. If $u$ is 0-essential then $v$ is 0 -special.

Proof. Suppose that $u$ and $v$ are 0-essential neighbours in the bipartite graph $G$. As $u v$ is a path, using Corollary 2.5 we find that

$$
\operatorname{mult}(0, G \backslash u v) \geq \operatorname{mult}(0, G)-1=\operatorname{mult}(0, G \backslash u),
$$

and therefore $v$ is not essential in $G \backslash u$. It follows from Lemma 3.8 that there is a 0-essential path $P$ in $G$ joining $u$ to $v$.

We now show that $P$ must have even length. From this it will follow that $P$ together with the edge $u v$ forms an odd cycle, which is impossible. From the definition of the matchings polynomial we see that $\operatorname{mult}(0, H)$ and $|V(H)|$ have the same parity for any graph $H$. As

$$
\operatorname{mult}(0, G \backslash P)=\operatorname{mult}(0, G)-1
$$

we deduce that $|V(G)|$ and $|V(G \backslash P)|$ have different parity and therefore $P$ has even length.

In the above proof we showed that a 0-essential path in a graph must have even length. Consequently no edge, viewed as a path of length one, can ever be 0-essential. It follows that $K_{1}$ is the only connected graph such that all paths are 0-essential. In general any graph which is minimal subject to its matchings polynomial having a particular zero $\theta$ will have the property that all its paths are $\theta$-essential.

Lemma 4.1 is not hard to prove without reference to the matchings polynomial. Note that it implies that in any bipartite graph there is a vertex which is covered by every maximal matching, and consequently that a bipartite graph with at least one edge cannot be 0-primitive. As noted by de Caen [2], this leads to a very simple inductive proof of König's lemma.

Our next result is a partial analog to the Edmonds-Gallai structure theorem. See, e.g., [8: Chapter 3.2]. 
4.2 Theorem. Let $\theta$ be a zero of $\mu(G, x)$ with non-zero multiplicity $k$ and let $a$ be a positive vertex in $G$. Then:

(a) if $u$ is essential in $G$ then it is essential in $G \backslash a$;

(b) if $u$ is positive in $G$ then it is essential or positive in $G \backslash a$;

(c) if $u$ is neutral in $G$ then it is essential or neutral in $G \backslash a$.

Proof. If $\operatorname{mult}(\theta, G \backslash u)=k-1$ and $\operatorname{mult}(\theta, G \backslash a)=k+1$, it follows by interlacing that $\operatorname{mult}(\theta, G \backslash a u)=k$. Hence $u$ is essential in $G \backslash a$. Now suppose that $u$ is positive in $G$. If $\operatorname{mult}(\theta, G \backslash a u) \geq k+1$ then $\theta$ has multiplicity at least $2 k+1$ as a zero of $p(x)$ where

$$
p(x):=\mu(G \backslash u, x) \mu(G \backslash a, x)-\mu(G, x) \mu(G \backslash a u, x) .
$$

By Lemma 2.4, the multiplicity of $\theta$ as a zero of $p(x)$ must be even. It follows that this multiplicity must be at least $2 k+2$ and hence that $\theta$ has multiplicity at least $2 k+2$ as a zero of $\mu(G, x) \mu(G \backslash a u, x)$. Therefore $\operatorname{mult}(\theta, G \backslash a u) \geq k+2$ and so, by interlacing, $\operatorname{mult}(\theta, G \backslash a u)=k+2$ and $u$ is positive in $G \backslash a$. If $\operatorname{mult}(\theta, G \backslash u a)=k+2$ and $u$ is neutral in $G$, then the multiplicity of $\theta$ as a zero of $p(x)$ is at least $2 k+1$ and therefore at least $2 k+2$, but this implies that $\theta$ is a zero of $\mu(G \backslash u, x) \mu(G \backslash a, x)$ with multiplicity at least $2 k+2$. Thus we conclude that $u$ is neutral or essential in $G \backslash a$.

We note that Theorem 4.2(a) holds even if $a$ is only neutral. If $a$ is neutral and $u$ is essential in $G$ but not in $G \backslash a$ then $\theta$ has multiplicity at least $2 k-1$ as a zero of (4.1) and so must have multiplicity at least $2 k$ as a zero of $\mu(G, x) \mu(G \backslash a u, x)$. Hence its multiplicity as a zero of $\mu(G \backslash u, x) \mu(G \backslash a, x)$ is at least $2 k$, which is impossible.

The following consequence of Theorem 4.2 and the previous remark was proved for trees by Neumaier. (See [9: Theorem 3.4(iii)].)

\subsection{Corollary. Any special vertex is positive.}

Proof. Suppose that $a$ is special in $G$, and that $u$ is a neighbour of $a$ which is essential in $G$. By part (a) of the theorem and the remark above, $u$ is essential in $G \backslash a$ and therefore, by Lemma $3.4, a$ is positive in $G$.

Lemma 3.7 implies that if $G$ is not $\theta$-critical then it contains a path, $P$ say, that is not essential. If we delete $P$ from $G$ then the multiplicity of $\theta$ as a zero of $\mu(G, x)$ cannot decrease. Hence we may successively delete 'inessential' paths from $G$, to obtain a graph $H$ such that $\operatorname{mult}(\theta, H) \geq \operatorname{mult}(\theta, G)$ and all paths in $H$ are essential. If $k=\operatorname{mult}(\theta, H)$ then, by Lemma 3.7 again, $H$ contains exactly $k$ critical components. The following result is a sharpening of this observation, since it implies that if $\operatorname{mult}(\theta, G)=k$ we may produce a graph with $k$ critical components by deleting $k$ vertex disjoint paths from $G$, 
4.4 Lemma. Let $G$ be a graph, let $\theta$ be a zero of $\mu(G, x)$ and let $u$ be a $\theta$-essential vertex of $G$. Suppose that there is a path in $\mathcal{P}(u)$ which is not $\theta$-essential. Then there is a path $P$ in $G$ starting at $u$ such that $\operatorname{mult}(\theta, G \backslash P)=\operatorname{mult}(\theta, G)$ and some component $C$ of $G \backslash P$ is critical. All vertices of $C$ are essential in $G$.

Proof. Suppose that there are paths in $\mathcal{P}(u)$ which are not essential, choose one of minimum length and call it $P$. Let $v$ be the end-vertex of $P$ other than $u$ and let $P^{\prime}$ be the path $P \backslash v$. Then $P^{\prime}$ is essential, hence

$$
\operatorname{mult}\left(\theta, G \backslash P^{\prime}\right)=\operatorname{mult}(\theta, G)-1
$$

and, as $P$ is not essential,

$$
\operatorname{mult}(\theta, G \backslash P) \geq \operatorname{mult}(\theta, G) .
$$

But we get $G \backslash P$ from $G \backslash P^{\prime}$ by deleting the single vertex $v$, therefore $\operatorname{mult}(\theta, G \backslash P)=$ mult $(\theta, G)$ and $v$ is positive in $G \backslash P^{\prime}$. Consequently, by Lemma 3.4, there is an essential vertex $u_{1}$ adjacent to $v$ in $\left(G \backslash P^{\prime}\right) \backslash v=G \backslash P$.

We now prove by induction on the number of vertices that, if the conditions of the lemma hold, then there is a path $P$ and a component $C$ of $G \backslash P$ as claimed and, further, there is a vertex $w$ in $C$ adjacent to the end-vertex of $P$ distinct from $u$ such that all paths in $C$ that start at $w$ are essential in $C$.

Let $H$ denote $G \backslash P$. If all paths in $H$ starting at $u_{1}$ are essential then, by Lemma 3.7, the component $C$ of $H$ that contains $u_{1}$ is critical. If $Q$ is a path in $C$ starting at $u_{1}$ then $\operatorname{mult}(\theta, C \backslash Q)<\operatorname{mult}(\theta, C)$; this implies that the path formed by the concatenation of $P$ and $Q$ is essential in $G$ and hence, by Lemma 3.3, that all vertices in $C$ are essential in $G$.

Thus we may suppose that there is a path in $H$ starting at $u_{1}$ that is not essential. Because $H$ has fewer vertices than $G$, we may assume inductively that there is a path $Q$ in $H$ starting at $u_{1}$ such that mult $(\theta, H)=\operatorname{mult}(\theta, H \backslash Q)$ and a critical component $C$ of $H \backslash Q$ that contains a neighbour $w$ of the end-vertex of $Q$ distinct from $u_{1}$. Further all the paths in $C$ that start at $w$ are essential.

Let $P Q$ denote the path formed by concatenating $P$ and $Q$. Then all claims of the lemma hold for $G, P Q, u$ and $C$.

The two results which follow provide a strengthening of the observation that the zeros of the matchings polynomial of a graph with a Hamilton path are simple.

4.5 Lemma. Suppose that $u$ and $v$ are adjacent vertices in $G$ such that $\mu(G \backslash u, x)$ and $\mu(G \backslash u v, x)$ have no common zero. Then $\mu(G, x)$ and $\mu(G \backslash u, x)$ have no common zero, and therefore both polynomials have have only simple zeros. 
Proof. Assume by way of contradiction that $\theta$ is a common zero of $\mu(G, x)$ and $\mu(G \backslash u, x)$. If mult $(\theta, G)>1$ then by Corollary 2.5 we see that $\theta$ is a zero of $\mu(G \backslash u, x)$ and $\mu(G \backslash u v, x)$. If mult $(\theta, G \backslash u)>1$ then mult $(\theta, G \backslash u v)>0$, by interlacing. Hence

$$
\operatorname{mult}(\theta, G)=\operatorname{mult}(\theta, G \backslash u)=1
$$

and so $u$ is a neutral vertex in $G$. It follows from Lemma 3.4 that no neighbour of $u$ can be essential in $G \backslash u$ and consequently $\operatorname{mult}(\theta, G \backslash u v)>0$.

A simple induction argument on the length of $P$ yields the following.

4.6 Corollary. Let $H$ be an induced subgraph of $G$ and suppose that there is a vertex $u$ in $H$ and a path $P$ in $G$ such that

$$
V(H) \cap V(P)=u, \quad V(H) \cup V(P)=V(G) .
$$

If $\mu(H, x)$ and $\mu(H \backslash u, x)$ have no common zero then all zeros of $G$ are simple.

Note that the path $P$ in this corollary does not have to be an induced path. One consequence of it is that if a graph has a Hamilton path then the zeros of its matchings polynomial are all simple. However this result shows that there will be many other graphs with all zeros simple.

\section{Eigenvectors}

Let $G$ be a graph with adjacency matrix $A=A(G)$. We view an eigenvector $f$ of $A$ with eigenvalue $\theta$ as a function on $V(G)$ such that

$$
\theta f(u)=\sum_{i \sim u} f(i)
$$

We denote the characteristic polynomial of $G$ by $\phi(G, x)$. (It is $\operatorname{defined~to~be~} \operatorname{det}(x I-$ $A(G))$.) We recall that for forests the characteristic and matchings polynomials are equal. Our first result follows from the proof of Theorem 5.2 in [3].

5.1 Lemma. Let $\theta$ be an eigenvalue of the graph $G$ and let $u$ be a vertex in $G$. Then the maximum value of $f(u)^{2}$ as $f$ ranges over the eigenvectors of $G$ with eigenvalue $\theta$ and norm one is equal to $\phi(G \backslash u, \theta) / \phi^{\prime}(G, \theta)$. 
5.2 Corollary (Neumaier [9: Theorem 3.4]). Let $T$ be a tree and let $\theta$ be a zero of its matchings polynomial. Then a vertex $u$ is essential if and only if there is an eigenvector $f$ of $T$ such that $f(u) \neq 0$.

5.3 Theorem. Let $T$ be a tree, let $\theta$ be a zero of $\mu(T, x)$ and let a be a vertex of $T$ which is not essential. Then a vertex is essential in $T \backslash a$ if and only if it is essential in $T$. Further, if $a$ is positive then it has an essential neighbour.

Proof. Let $W$ be the eigenspace of $T$ belonging to $\theta$ and let $W_{a}$ be the corresponding eigenspace of $T \backslash a$. Then $W_{a}$ is the direct sum of the eigenspaces of the component of $T \backslash a$ belonging to $\theta$ and $W$ is the subspace formed by the vectors $f$ such that

$$
\sum_{i \sim a} f(i)=0
$$

If $a$ is neutral then $W=W_{a}$ and so $T$ and $T \backslash a$ have the same essential vertices. If $a$ is positive then $W$ is a proper subspace of $W_{a}$, whence it follows that there are vectors in $W_{a}$ which are not zero on all neighbours of $a$. For each vector in $W_{a}$ there is an eigenvector in $W$ with the same support on $T \backslash a$. Hence $a$ has an essential neighbour and any vertex which is essential in $T \backslash a$ is also essential in $T$.

Theorem 5.3 is a strengthening of a result of Neumaier [9: Corollary 3.5]. Suppose that $T$ is a tree with exactly $s$ special vertices and $\operatorname{mult}(\theta, T)=k$. Then Theorem 5.3 together with Theorem 4.2 implies that we may successively delete the special vertices, obtaining a forest $F$ with no special vertices and $\operatorname{mult}(\theta, F)=k+s$. Hence any component of $F$ is either $\theta$-critical or does not have $\theta$ as a zero of its matchings polynomial. Therefore $F$ has exactly $k+s \theta$-critical components, and these components form an induced subgraph of $T$.

\section{Questions}

Many problems remain. Here are some.

(1) Must a positive vertex be special when $\theta \neq 0$ ? (If $\theta=0$ then all vertices which are not essential are positive.)

(2) What can be said of the graphs where every pair of vertices are joined by at least one essential path? (Or of the graphs with a vertex $u$ such that all vertices can be joined to $u$ by an essential path?)

(3) Must a $\theta$-primitive graph be $\theta$-critical? 
It might be interesting to investigate the case $\theta=1$ in depth.

\section{References}

[1] N. Biggs, Algebraic Graph Theory. (Cambridge U. P., Cambridge) 1974.

[2] D. de Caen, On a theorem of König on bipartite graphs, J. Comb. Inf. System Sci. 13 (1988) 127.

[3] C. D. Godsil, Matchings and walks in graphs, J. Graph Theory, 5, (1981) 285-297.

[4] C. D. Godsil and I. Gutman, On the theory of the matching polynomial, J. Graph Theory, 5 (1981), 137-144.

[5] C. D. Godsil, Real graph polynomials, in Progress in Graph Theory, edited by J. A. Bondy and U. S. R. Murty, (Academic Press, Toronto) 1984, pp. 281-293.

[6] C. D. Godsil, Algebraic Combinatorics. (Chapman and Hall, New York) 1993.

[7] O. J. Heilmann and E. H. Lieb, Theory of monomer-dimer systems, Commun. Math. Physics, 25 (1972), 190-232.

[8] L. Lovász and M. D. Plummer, Matching Theory. Annals Discrete Math. 29, (NorthHolland, Amsterdam) 1986.

[9] A. Neumaier, The second largest eigenvalue of a tree, Linear Algebra Appl. 48 (1982) $9-25$. 